\title{
Formulating Answerable Questions: Question Negotiation in Evidence-based Practice ${ }^{1,2}$
}

\author{
Lorie A. Kloda and Joan C. Bartlett
}

\begin{abstract}
Objective: This review explores the different question formulation structures proposed in the literature that may be helpful to librarians for conducting the reference interview and for teaching students and clinicians. Method: We present and compare several known question formulation structures identified in the health and social sciences literature. Discussion: Health and social care professionals should be made aware of the plurality of question formulation structures and their applicability to different fields of practice, as well as their utility for different types of questions within a field of practice.
\end{abstract}

\section{Introduction}

Librarians have important roles in assisting healthcare professionals and students in their information seeking. One of these roles is to help users identify and express their information needs clearly. This paper discusses the literature on formulating clinical questions in the context of health care. Linking question formulation to the practice of the reference interview, this paper first introduces Taylor's model of question negotiation to explain the first step of evidence-based practice. Various question formulation structures are reviewed, with examples provided as well as research on their usefulness. Finally, a discussion of the utility and applicability of question formulation structures for librarians, educators, and healthcare professionals is provided with suggestions for future research.

\section{Asking a question}

The basis of much of the interaction between librarians and information users (health professionals, researchers, students, and others) begins with questions that require answers. The question, a formal expression of an individual's information need, is the precursor and, in fact, the prerequisite for purposive information seeking to take place [1]. Librarians may expect users to approach them with fully formed and well-articulated questions as expressions of information needs that have been given much thought. In other words, librarians are prepared to assist or guide users in answering precise, unambiguous questions. Librarians are also, however, skilled in the art of the reference interview, a process by which the librarian interacts with the user to help the user articulate their information need and potentially narrow it to one or more questions. Much of what we think about the reference interview and its importance has its foundation in Robert Taylor's work on question negotiation [2].

Taylor's process of question negotiation emerged from his interest in the reference interaction or the reference interview. In the reference interaction, the person asking the question is in a difficult situation because, as Taylor explains, "one person tries to describe for another person not something he knows, but rather something he does not know" [2]. Taylor viewed such questions asked by users as dynamic, open-ended, and negotiable rather than static and unchanging.

There are three components to Taylor's model: the four types (or levels) of expression of information needs; the process model for prenegotiation decisions; and the five filters that questions go through during negotiation [3]. The first component is the most relevant to question formulation. The four levels of information needs or questions are: visceral need $(\mathrm{Q} 1)$, conscious need $(\mathrm{Q} 2)$, formalized need (Q3), and compromised need (Q4).

In Taylor's typology of needs, at the first level of question $(\mathrm{Q} 1)$, the user is not conscious of the need, and it remains vague and unexpressed; it is not even a question yet. The second level of question (Q2) is an acknowledged information need in the user's mind, but still ambiguous. At this level, Taylor suggests that the user may speak to

Lorie A. Kloda. ${ }^{3}$ McGill University Libraries, McLennan Library Building, 3459 McTavish Street, Montreal QC H3A 0C9.

Joan C. Bartlett. School of Information Studies, McGill University, 3661 Peel Street, Montreal QC H3A 1 X1.

${ }^{1}$ An earlier version of this paper was presented at the Canadian Health Libraries Association Annual Conference, Kingston, Ont., 7-11 June 2010.

${ }^{2}$ This article was peer reviewed.

${ }^{3}$ Corresponding author (e-mail: lorie.kloda@mcgill.ca). 
someone to help focus the question. The third level of question $(\mathrm{Q} 3)$ is the expressed information need, where the user may make a formal statement using concrete terms. The information need may be an interrogative statement, or it may be in the form of a declarative statement, such as "I would like to know more about...". At the fourth level (Q4) the question has been translated to accommodate a system (e.g., a bibliographic database, a search engine) or service for which they adjust their statement based on expectation. Thus, the question may be considered compromised.

Users who approach librarians for assistance may articulate their question anywhere between the second and fourth levels. That is, they may have trouble formalizing their information need precisely, or they may have altered the question to match a search strategy or their expectations of the librarian. Taylor argued that it is the intermediary (i.e., the librarian), during the reference encounter, who is often tasked with working back from an information need at the Q4 level to Q3 or even Q2 to find out what information is really needed.

Taylor's typology of four levels of question asking is a useful framework for librarians to determine the type of assistance they can offer a user during a reference encounter. Providing information to answer a user's question, articulated at the $\mathrm{Q} 3$ or $\mathrm{Q} 4$ level, by providing them with references or information may not actually satisfy their initial, conscious need. For a librarian to ensure they are truly assisting users, librarians need to ascertain information needs, which means that the user needs to be able to articulate it clearly. Simply stated, librarians must be interested not only in question answering but question asking as well.

Evidence-based practice (EBP) is the five-step process, best summarized in the Sicily Statement, that includes the following steps:

1. translation of uncertainty to an answerable question;

2. systematic retrieval of best evidence available;

3. critical appraisal of evidence for validity, clinical relevance, and applicability;

4. application of results in practice; and

5. evaluation of performance. [4]

Taylor's work on the process of question negotiation is especially relevant for health sciences librarians who are often tasked with teaching the first two steps of the EBP process and facilitating evidence use in general. Taylor's process of question negotiation is useful for understanding the levels of questioning the healthcare professional must use in the first step of the EBP process, supporting both the understanding and formulation of the question and the subsequent creation of a search query. By the time a healthcare professional approaches a librarian, they may have progressed through Taylor's first two levels of questions $(\mathrm{Q} 1, \mathrm{Q} 2)$ and possibly even the third and fourth levels (Q3, Q4).

A clinician struggling with the creation of an effective search strategy may benefit from assistance adapting the question to level Q4, whereas someone who is unsatisfied with search results may need to clarify their information need at the Q3 level. Librarians who teach clinicians and students to ask answerable questions can guide them through the four levels of questions. Many health sciences librarians do just that in teaching the first step of the EBP process to students and professionals in the health sciences. Research has yet to demonstrate, however, the effectiveness of such instruction on finding and using relevant research [5].

\section{Clinical question structures: method of selection for inclusion}

We reviewed question formulation structures proposed in published literature including journal articles, reports, and books. Structures in the health sciences and in social work were identified using iterative search strategies in social sciences and health sciences databases and through reference list harvesting of relevant papers. In addition, one structure was identified by word-of-mouth. Care was taken to identify and include any question formulation structure in health sciences or social work that makes mention of "clinical questions" or "evidence-based practice." A total of seven structures were identified in this manner and are described along with an example (from the original literature). We compared the various question formulation structures. While every attempt was made to be comprehensive in the inclusion of clinical question formulations, we present this as a selective list, as other structures may have been omitted inadvertently.

\section{The "anatomy" of answerable questions in health care}

In 1995, Richardson and colleagues first suggested a question formulation structure to assist physicians in creating an answerable question from an uncertainty arising during a patient encounter [6]. Their brief article proposed that formulating clinical questions according to their structure would help clinicians focus the ensuing literature search, and it would ensure that all relevant, high quality research be retrieved. The now widely used structure, known by its acronym PICO (problem, intervention, comparison, and outcome), is employed not only in medicine by evidence-based medicine proponents, but it has also been adopted in other areas of health care. Table 1 shows an example of a clinical question using the PICO structure.

A study by Dawes et al. [7] noted that the additional concept of "time" could be added to the PICO structure, and suggested that "results" was another important concept for patient management. They replaced the term "time" with "duration" (D) and the term "intervention" with "exposure" (E) and developed the PECODR (patient or problem, exposure, comparison, outcome, duration, and results) structure to include all the elements present in research studies. PECODR is intended to facilitate information retrieval by using the elements to match a clinical question with relevant abstracts in a bibliographic database. Table 1 shows an example of a clinical question employing the PECODR structure. 
Table 1. EBP question formulation examples from the literature.

\begin{tabular}{|c|c|c|}
\hline Method & Field used & Example \\
\hline $\begin{array}{l}\text { PECODR (patient or problem or } \\
\text { population, exposure, comparison, } \\
\text { outcome, duration, results) }\end{array}$ & Medicine & $\begin{array}{l}\text { P, 56-year-old man with hypertension; E, exposure } \\
\text { atenolol; C, placebo; O, cardiovascular event; } \\
\text { D, } 4.5 \text { years; R, number needed to treat of } 25\end{array}$ \\
\hline $\begin{array}{l}\text { PESICO (person, environments, } \\
\text { stakeholders, intervention, } \\
\text { comparison, outcome) }\end{array}$ & $\begin{array}{l}\text { Augmentative and } \\
\text { alternative communication } \\
\text { (AAC) }\end{array}$ & $\begin{array}{l}\text { A 4-year-old child with autism and some vocal imitation } \\
\text { skills in need of acquiring linguistic communication who } \\
\text { is placed in a segregated preschool (disabled peers) } \\
\text { seeking to move to an inclusive preschool (nondisabled } \\
\text { peers) and whose preschool teacher and speech-language } \\
\text { pathologist are primarily concerned with communication, } \\
\text { whereas his parents wish to enhance his speech as well. } \\
\text { Will the use of certain AAC approaches in comparison } \\
\text { with other AAC approaches enhance natural speech } \\
\text { production while increasing communication skills? }\end{array}$ \\
\hline
\end{tabular}

$\mathrm{PICO}+$ (patient, intervention, comparison, outcome, context, patient values and preferences)

COPES (client type and problem, what you might do about it, alternate course of action, what you want to accomplish)

ECLIPSE (expectation, client group, location, impact, professionals, service)
Occupational therapy

Social work

Health social care management, services, and policy

Medicine (oncology) guidelines
Not provided in the literature

For abused or neglected children placed in foster care by a protective service worker, which risk assessment measure will provide the greatest predictive accuracy to predict reabuse when children are placed back into the homes with their families?

There is a lack of continuity of care in my area for people with head injuries who are discharged from hospital to the community rehabilitation service. I would like to improve the discharge procedure to avoid this problem. The service involves both community health staff and social services. Has anyone else experienced similar problems and how have they overcome them?

What is appropriate cervical cancer screening for average risk women seen in primary care? interventions, professionals/patients, outcome, health care setting)
Since the PICO question formulation structure was suggested, several alternative structures have emerged in the health and social sciences literature (Table 1 shows a list with examples). In many cases these alternatives build on PICO, supplementing the four original elements of the structure with additional, and sometimes optional, elements for constructing answerable questions in various fields of practice. These alternatives grew out of the realization that not all clinical questions matched the medical focus of the PICO structure. Instead, these alternative structures included factors pertinent in other healthcare domains such as nursing or rehabilitation to better represent information needs that arise in practice.

For example, an alternative question formulation structure was put forward in the field of speech-language pathology, and more specifically augmentative and alternative communication. This structure, PESICO (person, environments, stakeholders, intervention, comparison, outcome), is considered more appropriate for formulating questions in that field as it includes elements that encourage details that may be relevant in finding answers $[8,9]$. PESICO incorporates all of the PICO elements, and adds "environments" (the contexts in which the problem occurs), and "stakeholders" (those with an interest in the outcome including. of course, the client and (or) patient).

In the field of occupational therapy, Bennett and Bennett [10] also made the argument that in addition to the elements in PICO, the client's context as well as values and preferences should be included when formulating questions. This suggestion of a "context" element, meant to represent environmental context and occupational factors, resembles the "environments" in PESICO. The "client's values and preferences" may also parallel "stakeholders" in PESICO, which includes the client's perspectives and attitudes. The similarity between Bennett and Bennett's proposed additional elements to those proposed 
by Schlosser et al. [9] suggests that the PESICO structure may be useful for formulating questions in rehabilitation professions besides speech-language pathology.

Additional question formulation structures have been proposed in other health and social care fields. One of these is the COPES (client-oriented, practical, evidence search) structure, used in the field of social work [11]. COPES is similar to PICO as it includes the same four elements that Gibbs [11] describes as "the client type and problem, what you might do, alternate course of action, and what you want to accomplish". The ECLIPSE (expectation, client group, location, impact, professionals, and service) structure [12], designed for management-related questions in health and social care, builds on the original PICO structure by specifying additional possible elements present in questions. Because ECLIPSE is designed to assist those in managing roles to formulate questions, the structure includes the elements of "location" (or setting) and "professionals" (for the type of practitioner) to help focus the question to match the evidence not only to the correct population of clients or patients but also to the specific groups of practitioners and an environment. Lastly, the PIPOH (population, intervention(s), professionals, outcomes, and health care setting/ context) structure, was designed by the ADAPTE Collaboration [13] to assist in the creation of practice guidelines. Though it uses different headings, PIPOH overlaps closely with ECLIPSE. Table 2 presents a summary of the similarities and differences between question formulation structures. A previous review of question structures provides a similar comparison, including those pertinent to library and information studies [14].

\section{Research on clinical question formulation}

Proponents of EBP have argued that formulating the original information need using the PICO structure assists clinicians in identifying research to resolve that information need [6]. While PICO is assumed to be useful for finding research, it has not been empirically demonstrated to actually represent physicians' information needs or uncertainties [15]. Huang et al. [15] concluded that few physicians' clinical questions followed the recommended PICO structure. The research did not identify why this might be the case, although one possible explanation is that the questions themselves did not include the elements suggested by PICO. It cannot be assumed, therefore, that forcing a question into PICO (or any structure) and then attempting to answer that question will necessarily resolve the clinician's original information need.

Over a decade ago, Booth et al. [16] investigated this very issue. They compared the use of a structured form to elicit the PICO elements from patrons' questions at the reference desk during the reference interview with a freeform approach. While the PICO structured form resulted in librarians conducting more precise searches, the questions rarely included elements other than "population" and "intervention" despite being specifically requested on the form. Interestingly, the researchers remarked that allowing a free-form question elicited details that enhanced the relevance of retrieved records, specifically, the inclusion of what was referred to as "context" or the purpose of the information request.

In a recent review of literature on emotions and information behaviour in health professionals, Fourie identified two themes relating to information needs [17]. The first of these themes was the difficulty surrounding the awareness and articulation of information needs. Fourie noted that several studies identified that health professionals (as with most people) have trouble recognizing their own information needs (that is, they are not aware of them), and this process can make it difficult to properly express the information need, or in fact, to recognize it as an information need at all. The second theme Fourie noted was uncertainty and anxiety. This was linked to an awareness of an information need. Individuals who identify information needs tend to express a level of uncertainty and sometimes stress or anxiety associated with that need. Understanding users' emotions pertaining to their information needs is important for librarians to offer assistance in acknowledging these needs and articulating them in a way that they might be answered.

\section{Utility and applicability of question formulation structures}

What these seven different question formulation structures offer are alternative methods for articulating infor-

Table 2. Comparison of EBP question formulation structures.

\begin{tabular}{|c|c|c|c|c|c|c|}
\hline PICO [6] & PECODR [7] & PESICO [8] & $\mathrm{PICO}+[10]$ & COPES [11] & ECLIPSE [12] & PIPOH [13] \\
\hline- & - & Stakeholders & $\begin{array}{l}\text { Patient values and } \\
\text { preferences }\end{array}$ & - & $\begin{array}{l}\text { Expectation } \\
\text { professionals }\end{array}$ & $\begin{array}{l}\text { Professional/ } \\
\text { patients }\end{array}$ \\
\hline Outcome & Outcome & Outcome & Outcome & $\begin{array}{l}\text { What you want to } \\
\text { accomplish }\end{array}$ & Impact & Outcome \\
\hline- & Duration & - & - & - & - & - \\
\hline- & Results & - & - & - & - & - \\
\hline
\end{tabular}


mation needs. Different professions (e.g., physicians vs. social workers) may benefit from different question formulations, as would individuals asking questions from differing perspectives. For example, a question from a management viewpoint or with the view of creating guidelines to inform many professionals could use ECLIPSE or PIPOH as a guide, whereas a clinician with a question in patient care may find PECODR or PESICO more applicable. It is noteworthy that allied health professions such as speech-language pathology and occupational therapy have both proposed question formulation structures that incorporate elements such as "stakeholders" or "client values" and "setting". This area merits further exploration. For example, do allied professionals have information needs that are more likely to include these elements?

Since its original publication in 1995 outlining the structure of the "well-built clinical question", librarians and educators in the health sciences have encouraged the use of PICO to help both formulate questions and create search queries as the first essential step in EBP. But PICO, and other structures, might best be thought of as guides or recommendations rather than strict formulations to be followed. Information needs should not be thought of in terms of restricted structures, as articulating and communicating them can already be difficult and linked to feelings of anxiety for the individual asking the question. Instead, question formulation structures can be used as guides for clinicians to articulating information needs at the Q3 level. Librarians can then use these structures as guides when assisting clinicians and students in the information seeking process and use the elements of the question to identify key concepts that will later be translated into search terms and criteria for assessing relevance. In this way, the process of question negotiation is enhanced. Clinical questions are not forced into structures, but they are translated from Q2 to Q3 as they are formalized and later compromised in Q4 into search terms. The information need, as articulated in Q2 or Q3 remains important as it contains the criteria by which any located evidence will be judged.

Despite the growing number of question formulation structures available to those in the health and social care fields, it cannot be assumed that these structures are exhaustive in describing the types of information needs that may arise in practice. It is to be expected that some information needs will not correspond to any structure, especially those for which qualitative or less traditional evidence may more effectively address the question. For this reason, librarians should recommend question formulation structures with some flexibility. As Booth et al. [16] demonstrated, insisting on structuring the information needs of users does not necessarily lead to better answers when librarians conduct literature searches. More research on whether this holds true for end-user searching is needed. In addition, it would be beneficial to investigate whether a link exists between articulating information needs using a question formulation structure and the likelihood of seeking (and finding) a relevant answer. Interfaces promoting asking questions using the PICO structure have already appeared (for example, PICO Linguist http://babelmesh.nlm.nih.gov/pico.php). A small pilot study has suggested that such an interface may be effective in improving search results [18], though more recent research has not found PICO structured searches to result in more effective searching [19].

Librarians and educators can encourage students and practitioners to formulate their questions using structures other than PICO if these structures offer more useful, relevant ways for thinking about and articulating their information needs. Librarians can also draw on question formulation structures during the reference interview process to assist in guiding the patron from a conscious need to a more formalized need, when such a structure is helpful. Health and social care professionals should be made aware of the plurality of the question formulation structures and their applicability to different fields of practice, as well as their utility for different types of questions within a field of practice.

Future research in this area is required to address whether question formulation structures are useful for librarians to employ in the context of the reference interview. In addition, research on how using question formulation structures may influence clinicians' information seeking behaviour would be of interest to librarians, educators, and providers of point-of-care research tools. Finally, studies identifying which available question formulation structures are the most useful for certain groups of health professionals and would be of value to librarians, educators, and others champions of EBP.

\section{References}

1. Wilson TD. Models in information behaviour research. J Doc. 1999;55(3):249-70. doi: 10.1108/EUM0000000007145.

2. Taylor RS. Question-negotiation and information seeking in libraries. Coll Res Libr. 1968;29(5):178-94.

3. Edwards PM. Taylor's question negotiation. In: Fisher KE, Erdelez S, McKechnie L, editors. Theories of Information Behavior. Medford, N.J.: American Society for Information Science and Technology by Information Today; 2005. p. $358-62$.

4. Dawes M, Summerskill W, Glasziou P, Cartabellotta A, Martin J, Hopayian K, et al. Sicily statement on evidencebased practice. BMC Med Educ. 2005;5.

5. Horsley T, O’Neill J, McGowan J, Perrier L, Kane G, Campbell C. Interventions to improve question formulation in professional practice and self-directed learning. Cochrane Database Syst Rev. 2010;5:CD007335. doi: 10.1002/ 14651858.CD007335.pub2.

6. Richardson SW, Wilson M, Nishikawa J, Hayward R. The well-built clinical question: a key to evidence-based decisions. ACP J Club.. 1995;123(3):A12-A13.

7. Dawes M, Pluye P, Shea L, Grad RM, Greenberg A, Nie J-Y. The identification of clinically important elements within medical journal abstracts: Patient - Population - Problem, Exposure - Intervention, Comparison, Outcome, Duration and Results (PECODR). Inform Prim Care. 2007;15(1):9-16.

8. Schlosser RW, O'Neil-Pirozzi T. Problem formulation in evidence-based practice and systematic reviews. Contemp Issues Commun Sci Disord. 2006;33:5-10. 
-9. Schlosser RW, Koul R, Costello J. Asking well-built questions for evidence-based practice in augmentative and alternative communication. J Commun Disord. 2007;40(3):225-38. doi: 10.1016/j.jcomdis.2006.06.008.

10. Bennett S, Bennett JW. The process of evidence-based practice in occupational therapy: informing clinical decisions. Aust Occup Ther J. 2000;47(4):171-80. doi: 10.1046/j.14401630.2000.00237.x.

11. Gibbs LE. Evidence-based practice for the helping professions. Toronto: Thomson Brooks Cole; 2003.

12. Wildridge V, Bell L. How CLIP became ECLIPSE: a mnemonic to assist in searching for health policy/manage ment information. Health Info Libr J. 2002;19(2):113-5. doi: 10.1046/j.1471-1842.2002.00378.x.

13. ADAPTE Collaboration. The ADAPTE process: resource toolkit for guideline adaptation. Version 2.0. 2009. http:// www.g-i-n.net/document-store/adapte-resource-toolkit-guide line-adaptation-version-2/view

14. Davies KS. Formulating the evidence based practice question: a review of the frameworks. Evid Based Libr Inform Practice. 2011;6(2):75-80.
15. Huang X, Lin J, Demner-Fushman D. Evaluation of PICO as a knowledge representation for clinical questions. In: $A M I A$ Symp Proc. 2006. pp. 359-63.

16. Booth A, O'Rourke AJ, Ford NJ. Structuring the pre-search reference interview: a useful technique for handling clinical questions. Bull Med Libr Assoc. 2000;88(3):239-47.

17. Fourie I. Learning from research on the information behaviour of healthcare professionals: a review of the literature 2004-2008 with a focus on emotion. Health Info Libr J. 2009;26(3):171-86.

18. Schardt C, Adams MB, Owens T, Keitz SA, Fontelo P. Utilization of the PICO framework to improve searching PubMed for clinical questions. BMC Med Inform Decis Mak. 2007;7:16.

19. Hoogendam A, Robbe PFDV, Overbeke AJPM. Comparing patient characteristics, type of intervention, control, and outcome (PICO) queries with unguided searching: a randomized controlled crossover trial. $J$ Med Libr Assoc. 2012;100:121-6. doi: 10.3163/1536-5050.100.2.010. 